\title{
Quantum renormalization group and holography
}

\author{
Sung-Sik Lee \\ Department of Physics \& Astronomy, McMaster University, \\ 1280 Main St. W., Hamilton ON L8S 4M1, Canada \\ Perimeter Institute for Theoretical Physics, \\ 31 Caroline St. N., Waterloo ON N2L 2Y5, Canada \\ E-mail: slee@mcmaster.ca
}

ABSTRACT: Quantum renormalization group scheme provides a microscopic understanding of holography through a general mapping between the beta functions of underlying quantum field theories and the holographic actions in the bulk. We show that the Einstein gravity emerges as a holographic description upto two derivative order for a matrix field theory which has no other operator with finite scaling dimension except for the energymomentum tensor. We also point out that holographic actions for general large $N$ matrix field theories respect the inversion symmetry along the radial direction in the bulk if the beta functions of single-trace operators are gradient flows with respect to the target space metric set by the beta functions of double-trace operators.

KEYwORDS: AdS-CFT Correspondence, Holography and condensed matter physics (AdS/CMT), Renormalization Group

ARXIV EPRINT: 1305.3908 
Renormalization group (RG) flow describes how short distance fluctuations modify coupling constants (coupling functions in general) as a system is probed at progressively larger length scales. Although RG provides a general framework for quantum field theories [1-3], it is of limited practical use for strongly coupled theories due to the fact that one has to keep track of a large (often infinite) set of operators.

AdS/CFT correspondence [4] provides an alternative way of organizing RG which is tractable for a certain set of strongly coupled quantum field theories. According to the dictionary of the conjecture [5,6], D-dimensional coupling functions of quantum field theories become dynamical variables in a $(D+1)$-dimensional bulk space. The radial direction in the bulk plays the role of the length scale in RG. The saddle point solution of an action in the bulk describes the evolution of the coupling functions along the radial direction, which can be interpreted as RG flow [7-9]. Despite this natural interpretation, the connection between holography and RG has been incomplete because the bulk fields are in general dynamical and quantum variables. They are dynamical in the sense that the bulk action includes two-derivative terms along the radial direction, and quantum because bulk fields have non-trivial quantum fluctuations. In conventional RG, on the contrary, coupling functions are non-dynamical and classical in the sense that they obey first-order beta functions, and an initial condition completely fixes the coupling functions at lower energy scales without any uncertainty.

A precise connection between holography and RG can be made via quantum renormalization group [10,11]. Unlike the conventional RG scheme, only a subset of operators is kept in quantum RG. The price one has to pay is to promote the coupling functions to dynamical fields. The partition function is given by a sum over all possible RG paths for the coupling functions of the operators in the subset. The weight for each path is determined by an action for the scale-dependent dynamical sources. In the context of matrix field theories, one needs to include only single-trace operators, although multi-trace operators are generated in the Wilsonian effective action [12, 13]. In quantum RG, double-trace operators generated at each step of coarse graining become kinetic terms for the sources of single-trace operators, allowing them to have non-trivial quantum fluctuations. The role of double-trace operators in holography was also emphasized in refs. [14, 15]. Quantum RG allows one to establish precise connections between the beta functions of quantum field theories and the bulk actions. In this paper, we show that quantum gravity can be derived from a matrix field theory via quantum RG.

Let us consider a large $N$ matrix field theory in the 't Hooft limit. We consider a set of primary single-trace operators $\left\{O_{n}\right\}$ constructed from a trace of products of microscopic matrix fields, $\Phi_{a}$. Any operator can be written as derivatives and multiplications of them. In conventional RG, one has to include not only the single-trace operators but also all multi-trace operators.

Quantum RG is formulated only in terms of the primary single-trace operators. The first step is to project fixed points to the subspace of the single-trace operators. In general, there exists no real fixed point on the subspace because multi-trace operators are generated out of single-trace operators under RG flow. However, one can have a projected fixed point. It refers to a theory at which RG flow is orthogonal to the subspace. In other words, a 


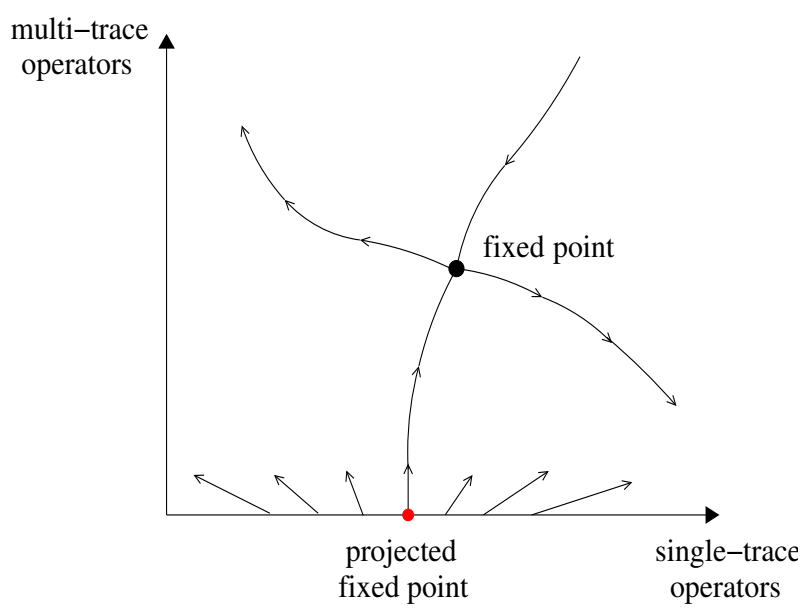

Figure 1. At fixed points, RG flow vanishes. At projected fixed points, only the projected RG flow along the subspace of single-trace operators vanishes.

projected fixed point is a fixed point under the RG flow projected onto the subspace of single-trace operators. This is illustrated in figure 1.

Let us consider a projected fixed point whose action $S_{0}[\Phi]$ is made of single-trace operators in the $D$-dimensional Minkowski space. To the theory, single-trace and multi-trace deformations can be added. The generating function is given by $Z=\int D \Phi e^{i\left(S_{0}+S_{1}+S_{2}\right)}$. Here the single-trace deformation is written as $S_{1}\left[O_{m} ; J^{m}\right]=N^{2} \sum_{m} \int d^{D} x J^{m}(x) O_{m}$ with spacetime dependent sources $J^{m}(x) . S_{2}\left[O_{m}, \mathcal{J}\right]$ is the multi-trace deformation which includes quadratic or higher order terms in $O_{m}$, where $\mathcal{J}$ represents sources for the multi-trace operators. We normalize the single-trace operators to be $O(1)$.

The multi-trace deformations can be removed by promoting the sources for the singletrace operators to dynamical fields. For this, we rewrite the generating function as

$$
\begin{aligned}
Z[J, \mathcal{J}]= & \int D \Phi \exp \left(i S_{0}+i S_{2}\left[-\frac{i}{N^{2}} \frac{\delta}{\delta J^{m}} ; \mathcal{J}\right]\right) \\
& \times \exp \left(i S_{1}\left[O_{m}, J^{m}\right]\right)
\end{aligned}
$$

where every $O_{m}(x)$ in $S_{2}$ is replaced by the functional derivative, $-\frac{i}{N^{2}} \frac{\delta}{\delta J^{m}(x)}$. We introduce a pair of auxiliary fields $j^{(0) m}, p_{m}^{(0)}$ for each single-trace operator $[10,11]$ to write

$$
\begin{aligned}
Z[J, \mathcal{J}]= & \int D j^{(0) m} D p_{m}^{(0)} D \Phi e^{i S_{0}+i N^{2} \int d^{D} x p_{m}^{(0)}\left(j^{(0) m}-J^{m}\right)} \\
& \times e^{i S_{2}\left[-i / N^{2} \delta / \delta j^{(0) m} ; \mathcal{J}\right]} e^{i S_{1}\left[O_{m} ; j^{(0) m}\right]} .
\end{aligned}
$$

Integrating $j^{(0) m}$ by part, the multi-trace terms are turned into a polynomial of $p_{m}^{(0)}$,

$$
\begin{aligned}
Z[J, \mathcal{J}]= & \int D j^{(0) m} D p_{m}^{(0)} e^{i N^{2} \int d^{D} x p_{m}^{(0)}\left(j^{(0) m}-J^{m}\right)} \\
& \times e^{i S_{2}\left[-p_{m}^{(0)} ; \mathcal{J}\right]} Z\left[j^{(0)}\right],
\end{aligned}
$$


where $Z\left[j^{(0)}\right]$ is the generating function with the single-trace deformation, $S_{1}\left[O_{m} ; j^{(0) m}\right]$. The original theory with multi-trace deformations is mapped into a theory with only singletrace operators whose sources are dynamical.

Now we focus on $Z\left[j^{(0)}\right]$ and apply renormalization group procedure. Under a coarse graining, high-energy modes are integrated out, and the UV-cut off is lowered by a factor of $e^{-d z}$. This renormalizes the deformation into $S_{1}\left[O_{m} ; j^{(0) m}\right]+\delta S$, where

$$
\begin{aligned}
\delta S\left[O_{n} ; j^{(0) n}\right]= & d z N^{2} \int d^{D} x\left\{\mathcal{L}_{c}\left(x ; j^{(0)}\right]\right. \\
& \left.-\beta^{m}\left(x ; j^{(0)}\right] O_{m}+\frac{G^{m n\{\mu\}}\left(x ; j^{(0)}\right]}{2} O_{m} \partial_{\{\mu\}} O_{n}\right\} .
\end{aligned}
$$

Here $f\left(x ; j^{(0)}\right]$ denotes a function that depends on $j^{(0)}(x)$ and their derivatives at position $x$. $\mathcal{L}_{c}\left(x ; j^{(0)}\right]$ is the Casimir energy that is generated out of integrating out high-energy modes. It can be viewed as the quantum correction to the identity operator. $\beta^{m}\left(x ; j^{(0)}\right]$ represents the beta functional for the single-trace operators. ${ }^{1}$ Because $S_{0}$ is a projected fixed point, $\beta^{m}$ (but not $G^{m n\{\mu\}}$ ) vanishes at $j^{(0)}=0$, and can be expanded as $\beta^{m}\left(x ; j^{(0)}\right]=\Delta_{n}^{m} j^{(0) n}+$ $O\left(\left(j^{(0)}\right)^{2}\right)$. If there exists a single-trace operator with a scaling dimension $\Delta_{O}$ under the projected RG flow, $\Delta_{n}^{m}$ has an eigenvalue $\Delta_{O}-D . G^{m n\{\mu\}}\left(x ; j^{(0)}\right]$ is the source for doubletrace operators generated from quantum corrections. $\{\mu\}$ denotes a series of derivative, $\left(\partial_{\mu_{1}} \partial_{\mu_{2}} \ldots\right)$. No higher-trace operators are generated to the order of $d z$. Following the same steps as in eqs. (1)-(3), another set of auxiliary fields are introduced to remove the double-trace operators as

$$
\begin{aligned}
Z\left[j^{(0)}\right]= & \int D j^{(1) n} D p_{n}^{(1)} e^{i N^{2} \int d^{D} x p_{n}^{(1)}\left(j^{(1) n}-j^{(0) n}\right)} \\
& \times e^{i \delta S\left[-p_{n}^{(1)} ; j^{(0) n}\right]} Z\left[j^{(1)}\right] .
\end{aligned}
$$

It can be explicitly checked that $S_{1}+\delta S$ is reproduced as the auxiliary fields are integrated out.

By iterating these steps, one can write the generating function as a functional integration of the auxiliary fields introduced at each step of coarse graining,

$$
Z\left[j^{(0)}\right]=\int \Pi_{l=1}^{L} D j^{(l) n} D p_{n}^{(l)} e^{i S_{B}} Z\left[j^{(L)}\right],
$$

where

$$
\begin{aligned}
S_{B}= & N^{2} d z \sum_{l=1}^{L} \int d^{D} x\left\{p_{n}^{(l)} \frac{j^{(l) n}-j^{(l-1) n}}{d z}\right. \\
& \left.+\delta S\left[-p_{n}^{(l)} ; j^{(l-1) n}\right]\right\}
\end{aligned}
$$

\footnotetext{
${ }^{1}$ Descendants of $O_{m}$ can be removed by integration by part.
} 
In the $d z \rightarrow 0$ limit, $j^{(l) n}(x), p_{n}^{(l)}(x)$ become $(D+1)$-dimensional fields, $j^{n}(x, z), p_{n}(x, z)$ with $z=l d z$. The bulk action is written as

$$
\begin{aligned}
& S_{B}=N^{2} \int_{0}^{z^{*}} d z \int d^{D} x\left\{p_{n}\left(\partial_{z} j^{n}\right)+\mathcal{L}_{c}(x ; j]\right. \\
& \left.+\beta^{m}(x ; j] p_{m}+\frac{G^{m n\{\mu\}}(x ; j]}{2} p_{m} \partial_{\{\mu\}} p_{n}\right\},
\end{aligned}
$$

where $z^{*}=L d z$ is an IR scale at which we stop the RG procedure. Without loss of generality, we can take $z^{*}=\infty$. If the scale $z$ is interpreted as a 'time', the dynamical source $j$ and the operator field $p$ become canonically conjugate to each other. The Casimir energy, $\mathcal{L}_{c}$ becomes the potential 'energy' of the source fields $j$, and the quadratic term in $p$ becomes the kinetic 'energy'. The fact that sources become dynamical in the bulk is also natural from the point of view of string theory in that dynamical closed strings provide sources for open strings which define field theory on D-branes [16].

Now we consider a scale-reversal (SR) transformation: $j^{n}(x, z) \rightarrow j^{n}\left(x, z^{*}-z\right)$, $p_{n}(x, z) \rightarrow-p_{n}\left(x, z^{*}-z\right)$. Since RG flow is irreversible, one naively expects that the bulk action should always break the SR symmetry. However, SR symmetric bulk actions can still describe irreversible RG flows because of a boundary at the UV cut-off scale (and also an IR boundary if there is an IR cut-off as well), which explicitly break the SR symmetry. In the bulk action, only the third term in eq. (8) breaks the symmetry. It turns out that the SR-symmetry breaking term in the bulk can be removed if there exists a $D$-dimensional $c$-functional $c[j(x)]$ which generates the projected RG flow of single-trace operators as a gradient flow,

$$
\beta^{m}(x ; j]=G^{m n\{\mu\}}(x ; j] \partial_{\{\mu\}} \frac{\delta c[j]}{\delta j^{n}(x)},
$$

where $G^{m n\{\mu\}}(x ; j]$ plays the role of a 'super-metric' in the space of single-trace operators. In this case, the conjugate momentum can be shifted as $p^{n} \rightarrow p^{n}-\frac{\delta c}{\delta j_{n}}$, and the SR odd term becomes a boundary terms, $N^{2}\left(c[j(x, 0)]-c\left[j\left(x, z^{*}\right)\right]\right)$. Then the bulk action is written as $S_{B}=N^{2} \int d z\left\{\int d^{D} x p_{n}\left(\partial_{z} j^{n}\right)-H\right\}$, where the 'Hamiltonian' is given by

$$
H=-\frac{1}{2} \boldsymbol{p}^{T} \cdot \mathbf{G} \cdot \boldsymbol{p}+\frac{1}{2} \boldsymbol{\beta}^{T} \cdot \mathbf{G}^{-1} \cdot \boldsymbol{\beta}-\int d^{D} x \mathcal{L}_{c}
$$

which respects the SR symmetry. Here $\boldsymbol{p}, \boldsymbol{\beta}$ are understood as vectors whose indices run over $m$ and $x$ ( $\boldsymbol{p}^{T}, \boldsymbol{\beta}^{T}$ are their transposes) and $\mathbf{G}$ is a matrix. The second term on the r.h.s. of eq. (10) along with the quadratic term of the Casimir energy determines the mass of the source fields. To the quadratic order in $j$, it becomes $\frac{1}{2} \boldsymbol{\beta}^{T} \cdot \mathbf{G}^{-1} \cdot \boldsymbol{\beta}=\frac{1}{2} \mathbf{j}^{T} \cdot \boldsymbol{\Delta}^{T} \cdot \mathbf{G}^{-1} \cdot \boldsymbol{\Delta} \cdot \mathbf{j}$. This explains why the scaling dimension of an operator determines the mass of the corresponding field in the bulk. Note that the mass is positive when $\mathbf{G}$ is positive. In this case, the kinetic term has the 'wrong' sign. This implies that the radial direction is space-like not time-like. 
If $G^{m n\{\mu\}}(x ; j] \partial_{\{\mu\}}$ is positive semidefinite, the $c$-functional that satisfies eq. (9) decreases monotonically under the projected RG flow,

$$
\begin{aligned}
\frac{d c[j]}{d z} & =-\int d x \frac{\delta c[j]}{\delta j^{m}(x)} \beta^{m}(x ; j] \\
& =-\int d x \frac{\delta c[j]}{\delta j^{m}(x)} G^{m n\{\mu\}}(x ; j] \partial_{\{\mu\}} \frac{\delta c[j]}{\delta j^{n}(x)} \leq 0 .
\end{aligned}
$$

It is interesting to note that the SR symmetry of the bulk action is related to the monotonicity of the projected RG flow. When the RG flow is a gradient flow, the irreversibility parameterized by the $c$-functional depends only on the initial and final points, but not on the path connecting the two end points. That is why the SR symmetry breaking term in the bulk action can be transferred to the boundary terms.

As a concrete example, let we consider a projected fixed point of a matrix field theory where the single-trace energy-momentum tensor is the only operator that has finite scaling dimension under projected RG flow. All other single-trace operators have infinite scaling dimensions and they instantly die out if they are generated under RG flow. Therefore a general single-trace action is completely specified by background metric. Consequently, the only operators that arise in the full un-projected RG flow are the single-trace energy-momentum tensor and multi-trace operators made of the energy-momentum tensor. Although it is not clear whether such a matrix field theory exists, we proceed with the assumption that it exists to illustrate how quantum gravity emerges via quantum RG in a simple setting. For more general field theories, one has to include more operators [11], but the generalization is straightforward.

The generating function is written as $Z\left[g^{(0)}\right]=\int D \Phi e^{i S_{1}\left[\Phi ; g^{(0)}(x)\right]}$, where $\Phi$ represents underlying microscopic degrees of freedom, and $g_{\mu \nu}^{(0)}(x)$ with $\mu, \nu=0,1, \ldots,(D-1)$ is a $D$-dimensional background metric with signature $(-,+,+, \ldots,+)$. It is assumed that the regularization scheme respects the $D$-dimensional diffeomorphism invariance. $S_{1}$ includes only single-trace operators and is $O\left(N^{2}\right)$ in the 't Hooft limit. The energy-momentum tensor, normalized to be $O(1)$, is given by $T^{\mu \nu}\left(x ; g^{(0)}\right]=\frac{1}{N^{2} \sqrt{\left|g^{(0)}\right|}} \frac{\delta S_{1}}{\delta g_{\mu \nu}^{(0)}(x)}$. Under a coarse graining, the action is modified by a quantum correction $\delta S^{(1)^{\prime}}$ which includes the Casimir energy, the single-trace energy-momentum tensor and double-trace operators constructed from $T^{\mu \nu}$. The new effective action $S_{1}\left[\Phi ; g^{(0)}(x)\right]+\delta S^{(1)^{\prime}}$ should reproduce the exact same generating function,

$$
\begin{aligned}
Z\left[g^{(0)}\right]= & \int D g_{\mu \nu}^{(1)} D \pi^{(1) \mu \nu} D \Phi e^{i N^{2} \int d^{D} x \pi^{(1) \mu \nu}\left(g_{\mu \nu}^{(1)}-g_{\mu \nu}^{(0)}\right)} \\
& \times e^{i \delta S^{(1)^{\prime}}\left[i / N^{2} \delta / \delta g_{\mu \nu}^{(1)}, g^{(0)}\right]} e^{i S_{1}\left[\Phi ; g^{(1)}\right]},
\end{aligned}
$$

where the quantum correction is expressed in terms of functional derivative with respect to auxiliary fields as in eq. (2),

$$
\begin{aligned}
\delta S^{(1)^{\prime}}= & d z \int d^{D} x n^{(1) z}(x)\left\{N^{2} \sqrt{\left|g^{(0)}\right|}\left(-C_{0}+C_{1}{ }^{D} \mathcal{R}\left(x ; g^{(0)}\right]\right)+i A_{\mu \nu}\left(x ; g^{(0)}\right] \frac{\delta}{\delta g_{\mu \nu}^{(1)}(x)}\right. \\
& \left.-\frac{B_{\mu \nu ; \rho \sigma}\left(x ; g^{(0)}\right]}{2 N^{2}} \frac{\delta}{\delta g_{\mu \nu}^{(1)}(x)} \frac{\delta}{\delta g_{\rho \sigma}^{(1)}(x)}+\ldots\right\} .
\end{aligned}
$$


Here we adopt a local RG scheme $[11,17]$ where the length scale is increased in a spacetime dependent way: $n^{(1) z}(x)$ is a local speed of coarse graining. $C_{0}, C_{1}{ }^{D} \mathcal{R}$ are the first two leading order terms of the Casimir energy in the derivative expansion, where ${ }^{D_{\mathcal{R}}}$ is the $D$-dimensional Ricci scalar $[18,19] . A_{\mu \nu}$ represents the warping factor of the $D$ dimensional spacetime. $B_{\mu \nu, \rho \sigma}$ represents the source of the double-trace operator, $T^{\mu \nu} T^{\rho \sigma}$ that is generated under coarse graining. From dimensional ground, we expect $C_{0} \sim a^{-D}$, $C_{1} \sim a^{-D+2}, A_{\mu \nu} \sim 1$ and $B_{\mu \nu ; \rho \sigma} \sim a^{D}$, where $a$ is a short-distance cut-off scale, the only scale in the theory. The ellipsis represents higher derivative terms in the Casimir energy and the contribution of the operators that involve at least one derivative, such as $\left(\nabla_{\alpha_{1}}^{(1)} \nabla_{\alpha_{2}}^{(1)} \ldots\right) \frac{\delta}{\delta g_{\mu \nu}^{(1)}(x)}, \frac{\delta}{\delta g_{\mu \nu}^{(1)}(x)}\left(\overleftarrow{\nabla}_{\alpha_{1}}^{(1)} \vec{\nabla}_{\alpha_{2}}^{(1)} \ldots\right) \frac{\delta}{\delta g_{\rho \sigma}^{(1)}(x)}$, where $\nabla^{(1)}$ is the covariant derivative with respect to the metric $g_{\mu \nu}^{(1)}$. The higher derivative terms are suppressed by additional powers of $a$.

In the local RG, $n^{(1) z}$ is a gauge freedom which controls the local speed of coarse graining. One can introduce another gauge freedom by using the fact that $Z\left[g^{(1)}\right]$ is invariant under the $D$-dimensional diffeomorphism, $Z\left[g_{\mu \nu}^{(1)}\right]=Z\left[g_{\mu \nu}^{(1)}+d z\left(\nabla_{\mu}^{(1)} n_{\nu}^{(1)}+\nabla_{\nu}^{(1)} n_{\mu}^{(1)}\right)\right]$, where $d z n^{(1) \mu}$ is an infinitesimal shift of the $D$-dimensional coordinates for the low-energy field with respect to the coordinates of the high-energy field. This leads to

$$
\begin{aligned}
Z\left[g^{(0)}\right]= & \int D g_{\mu \nu}^{(1)} D \pi^{(1) \mu \nu} D \Phi e^{i N^{2} \int d^{D} x \pi^{(1) \mu \nu}\left(g_{\mu \nu}^{(1)}-g_{\mu \nu}^{(0)}\right)} \\
& e^{i \delta S^{(1)^{\prime}}\left[i / N^{2} \delta / \delta g_{\mu \nu}^{(1)} ; g^{(0)}\right]} e^{i \delta S^{(1)^{\prime \prime}}\left[i / N^{2} \delta / \delta g_{\mu \nu}^{(1)}\right]} e^{i S_{1}\left[\Phi ; g^{(1)}\right]}
\end{aligned}
$$

where $\delta S^{(1)^{\prime \prime}}=-i d z \int d^{D} x\left(\nabla_{\mu}^{(1)} n_{\nu}^{(1)}+\nabla_{\nu}^{(1)} n_{\mu}^{(1)}\right) \frac{\delta}{\delta g_{\mu \nu}^{(1)}(x)}$. Integrating $g_{\mu \nu}^{(1)}$ by part, $\delta S^{(1)^{\prime}}+$ $\delta S^{(1)^{\prime \prime}}$ becomes a quadratic polynomial of $\pi^{(1) \mu \nu}$. Repeating these steps, the generating function is written as

$$
Z\left[g^{(0)}\right]=\int \prod_{l=1}^{L}\left[D g_{\mu \nu}^{(l)}(x) D \pi^{(l) \mu \nu}(x)\right] \quad e^{i S_{B}} Z\left[g^{(L)}(x)\right],
$$

where the bulk action, to the linear order of $d z$, becomes

$$
\begin{aligned}
S_{B}= & N^{2} d z \sum_{l=1}^{L} \int d^{D} x\left\{\pi^{(l) \mu \nu} \frac{g_{\mu \nu}^{(l)}-g_{\mu \nu}^{(l-1)}}{d z}\right. \\
& +2 n^{(l) \mu}(x) \nabla^{(l) \nu} \pi_{\mu \nu}^{(l)}+n^{(l) z}(x, z)\left[\sqrt { | g ^ { ( l - 1 ) } | } \left(-C_{0}\right.\right. \\
& \left.+C_{1}{ }^{D} \mathcal{R}\left(x, g^{(l-1)}\right]\right)+A_{\mu \nu}\left(x ; g^{(l-1)}\right] \pi^{(l) \mu \nu} \\
& \left.\left.+\frac{B_{\mu \nu ; \rho \sigma}\left(x ; g^{(l-1)}\right]}{2} \pi^{(l) \mu \nu} \pi^{(l) \rho \sigma}+\ldots\right]\right\} .
\end{aligned}
$$

In the $d z \rightarrow 0$ limit, the metric and the conjugate field become $(D+1)$-dimensional fields. In order to make the radial coordinate more symmetric with the $D$-dimensional coordinate $x$, we introduce a dimensionful radial coordinate $x^{D}=a e^{l d z}$, and define $(D+1)$ dimensional coordinate as $X=\left(x, x^{D}\right)$. The dimensionless lapse and shift functions are 
defined as $N^{\mu}(X) \equiv n^{(l) \mu}(x) / x^{D}$ and $N^{D}(X) \equiv n^{(l) z}(x) a / x^{D}$. Then the bulk theory takes the form of a constrained Hamiltonian system for the metric and its conjugate momentum [20],

$$
S_{B}=N^{2} \int d X^{D+1}\left\{\pi^{\mu \nu}\left(\partial_{D} g_{\mu \nu}\right)-N^{\mu} \mathcal{H}_{\mu}-N^{D} \mathcal{H}\right\},
$$

where the momentum and Hamiltonian constraints are given by

$$
\begin{aligned}
\mathcal{H}_{\mu}= & -2 \nabla^{\nu} \pi_{\mu \nu}, \\
\mathcal{H}= & -\gamma \sqrt{|g|}\left(-\Lambda_{0}+{ }^{D} \mathcal{R}\right)-\beta_{\mu \nu} \pi^{\mu \nu} \\
& -\frac{\mathcal{G}_{\mu \nu ; \rho \sigma}}{2} \pi^{\mu \nu} \pi^{\rho \sigma}+\ldots
\end{aligned}
$$

with $\Lambda_{0}=C_{0} / C_{1}, \gamma=C_{1} / a, \beta_{\mu \nu}=A_{\mu \nu} / a$, and $\mathcal{G}_{\mu \nu ; \rho \sigma}=B_{\mu \nu ; \rho \sigma} / a$. The most general forms of the warping factor and the super-metric which are consistent with the $D$-dimensional diffeomorphism invariance are

$$
\begin{aligned}
\beta_{\mu \nu} & =\beta g_{\mu \nu}, \\
\mathcal{G}_{\mu \nu ; \rho \sigma} & =\frac{\alpha}{\sqrt{|g|}}\left(g_{\mu \rho} g_{\nu \sigma}-\lambda g_{\mu \nu} g_{\rho \sigma}\right)
\end{aligned}
$$

to the leading order in $a$. For unitary theories, $\alpha, \beta, \gamma, \Lambda_{0}$ are real, and their values depend on the matter content [19]. Here we focus on the case with $\alpha, \gamma>0$. In the bulk action, these parameters always appear in combination with $N^{z}$. Therefore one can choose $N^{z}$ to normalize one of the parameters. This freedom stems from the fact that only relative speeds of RG flows for different operators matter. One often chooses $N^{z}$ to fix the warping factor as $\beta=2 / a$ [17]. Here we make an alternative choice such that $\frac{\alpha}{2}=\frac{1}{\gamma} \equiv 2 \kappa^{2} \sim a^{D-1}$. As expected, the warping term, $\beta_{\mu \nu} \pi^{\mu \nu}$ breaks the SR symmetry. However, $\beta_{\mu \nu}$ can be written as a gradient flow $\beta_{\mu \nu}=\mathcal{G}_{\mu \nu, \rho \sigma} \frac{\delta c}{\delta g_{\rho \sigma}(x)}$ with $c=-\frac{\beta}{2 \kappa^{2}(D \lambda-1)} \int d^{D} x \sqrt{|g|}$. The SR-symmetry breaking term is traded with a cosmological constant $\frac{1}{2} \frac{\delta c}{\delta g_{\mu \nu}} \mathcal{G}_{\mu \nu ; \rho \sigma} \frac{\delta c}{\delta g_{\rho \sigma}}$ in the bulk and the surface tension term $N^{2}\left(c\left[g\left(x, x^{D}=a\right)\right]-c\left[g\left(x, x^{D}=\infty\right)\right]\right)$ at the boundaries.

In order to fix $\lambda$, we note that $\mathcal{H}=0$ and $\mathcal{H}^{\mu}=0$ on shell because the generating function is independent of the choice of $\left\{N^{\mu}(X), N^{D}(X)\right\}$ : choice of different RG prescriptions is a pure gauge freedom which does not affect the generating function. They have to be of first class because the constraint equations $\mathcal{H}=0, \mathcal{H}^{\mu}=0$ are satisfied on shell at any $X$ in the bulk for any choice of $\left\{N^{\mu}(X), N^{D}(X)\right\}$ [11]. The fact that they should form first-class constraints, which holds at each order in $a$, forces $\lambda=\frac{1}{D-1}$. In $D=3$, this was shown in ref. [21, 22]. It is straightforward to extend the result to general dimensions.

Once the canonical momentum is integrated out, one obtains the $(D+1)$-dimensional Einstein gravity upto the two derivative terms,

$$
S_{B}=\frac{N^{2}}{2 \kappa^{2}} \int d^{D+1} X \sqrt{|G|}\left(-\Lambda+{ }^{(D+1)} \mathcal{R}+\ldots\right) .
$$

Here $G_{M N}$ with $M, N=0,1,2, \ldots, D$ is the $(D+1)$-dimensional metric constructed from $g_{\mu \nu}, N^{\mu}$ and $N^{D}$ with signature $(-,+,+, \ldots,+)$. The signature of the radial direction 
is determined by the sign of $\alpha .^{2} \Lambda=\Lambda_{0}-\frac{D(D-1) \beta^{2}}{4}$ is the cosmological constant. $\Lambda_{0}$ is determined by the vacuum energy density of the field theory per unit RG length scale, that is, the contribution from the modes integrated between the length scale $a$ and $a e^{d z}$. If $\Lambda_{0}=0$, which holds for supersymmetric theories, the cosmological constant is negative as it is solely determined by the warping factor. In this case, one naturally obtains AdS in the bulk. The Newton constant is $G_{N} \sim \kappa^{2} / N^{2}$, where $\kappa^{2}$ is given by the ratio between the beta function of the double-trace energy-momentum tensor and the coefficient of ${ }^{D} \mathcal{R}$ in the Casimir energy. The ellipsis in eq. (20) denotes terms that have more than two derivatives.

If the underlying field theory does not have any other operator except for the energymomentum tensor, the higher derivative terms in eq. (20) can have more than two derivatives only along the field theory direction but not along the radial direction. For example, the bulk action may include $\left({ }^{D} \mathcal{R}\right)^{2}$ contributed from the Casimir energy. This would imply that the bulk action for the idealized field theory is anisotropic beyond the two derivative order. In reality, there are many other operators besides the energy-momentum tensor. As a result, one needs to include other fields in the bulk, which generate higher derivative terms for the metric in all directions once integrated out.

Generically, $\Lambda, \kappa^{2}$ and the coefficients of the higher derivative terms will be determined by the UV cut-off scale and the spectrum of operators for the boundary quantum field theory. One obtains a weakly curved spacetime in the bulk if the cosmological constant is smaller than the scales associated with the higher derivative terms, as is the case for the $\mathcal{N}=4$ super Yang-Mills theory in the strong coupling limit. It will be of great interest to understand the precise condition under which the weakly curved spacetime emerges in the bulk.

Finally, we comment on holographic duals for (ungauged) vector models [23]. Once multi-trace operators are traded with dynamical sources in the first step of $R G$ as in eq. (3), the resulting single-trace action remains quadratic in the following steps [24]. Because multi-trace operators are not generated, the bulk action is linear in the conjugate momenta [25] except at the UV boundary. The conjugate momenta remain as Lagrangian multipliers which enforce the usual first order beta function as constraints in the bulk. Therefore there is no quantum fluctuation in the RG path for any $N$.

We thank Hong Liu, Yu Nakayama, Joao Penedones, Joe Polchinski, Renate Loll, Sang-Jin Sin and Tadashi Takayanagi for helpful comments. This research was supported by ERA, NSERC and the Templeton Foundation. Research at the Perimeter Institute is supported in part by the Government of Canada and by the Province of Ontario.

Open Access. This article is distributed under the terms of the Creative Commons Attribution License (CC-BY 4.0), which permits any use, distribution and reproduction in any medium, provided the original author(s) and source are credited.

\footnotetext{
${ }^{2}$ If $\alpha$ was negative, the radial direction would be time-like.
} 


\section{References}

[1] K. Wilson, The renormalization group and critical phenomena, Rev. Mod. Phys. 55 (1983) 583 [INSPIRE].

[2] J. Polchinski, Renormalization and Effective Lagrangians, Nucl. Phys. B 231 (1984) 269 [INSPIRE].

[3] C. Wetterich, Exact evolution equation for the effective potential, Phys. Lett. B 301 (1993) 90 [INSPIRE].

[4] J.M. Maldacena, The Large-N limit of superconformal field theories and supergravity, Adv. Theor. Math. Phys. 2 (1998) 231 [Int. J. Theor. Phys. 38 (1999) 1113] [hep-th/9711200] [INSPIRE].

[5] S. Gubser, I.R. Klebanov and A.M. Polyakov, Gauge theory correlators from noncritical string theory, Phys. Lett. B 428 (1998) 105 [hep-th/9802109] [INSPIRE].

[6] E. Witten, Anti-de Sitter space and holography, Adv. Theor. Math. Phys. 2 (1998) 253 [hep-th/9802150] [INSPIRE].

[7] E.T. Akhmedov, A Remark on the AdS/CFT correspondence and the renormalization group flow, Phys. Lett. B 442 (1998) 152 [hep-th/9806217] [InSPIRE].

[8] J. de Boer, E.P. Verlinde and H.L. Verlinde, On the holographic renormalization group, JHEP 08 (2000) 003 [hep-th/9912012] [INSPIRE].

[9] K. Skenderis, Lecture notes on holographic renormalization, Class. Quant. Grav. 19 (2002) 5849 [hep-th/0209067] [INSPIRE]; references there-in.

[10] S.-S. Lee, Holographic description of large-N gauge theory, Nucl. Phys. B 851 (2011) 143 [arXiv: 1011.1474] [INSPIRE].

[11] S.-S. Lee, Background independent holographic description : From matrix field theory to quantum gravity, JHEP 10 (2012) 160 [arXiv:1204.1780] [INSPIRE].

[12] C. Becchi, S. Giusto and C. Imbimbo, The Wilson-Polchinski renormalization group equation in the planar limit, Nucl. Phys. B 633 (2002) 250 [hep-th/0202155] [InSPIRE].

[13] C. Becchi, S. Giusto and C. Imbimbo, The Renormalization of noncommutative field theories in the limit of large noncommutativity, Nucl. Phys. B 664 (2003) 371 [hep-th/0304159] [INSPIRE].

[14] I. Heemskerk and J. Polchinski, Holographic and Wilsonian Renormalization Groups, JHEP 06 (2011) 031 [arXiv: 1010.1264] [INSPIRE].

[15] T. Faulkner, H. Liu and M. Rangamani, Integrating out geometry: Holographic Wilsonian $R G$ and the membrane paradigm, JHEP 08 (2011) 051 [arXiv:1010.4036] [INSPIRE].

[16] E. Kiritsis, Lorentz violation, Gravity, Dissipation and Holography, JHEP 01 (2013) 030 [arXiv: 1207.2325] [INSPIRE].

[17] H. Osborn, Weyl consistency conditions and a local renormalization group equation for general renormalizable field theories, Nucl. Phys. B 363 (1991) 486 [INSPIRE].

[18] A. Sakharov, Vacuum quantum fluctuations in curved space and the theory of gravitation, Sov. Phys. Dokl. 12 (1968) 1040 [INSPIRE].

[19] M. Visser, Sakharov's induced gravity: A Modern perspective, Mod. Phys. Lett. A 17 (2002) 977 [gr-qc/0204062] [INSPIRE]; references there-in. 
[20] R.L. Arnowitt, S. Deser and C.W. Misner, Dynamical Structure and Definition of Energy in General Relativity, Phys. Rev. 116 (1959) 1322 [INSPIRE].

[21] D. Blas, O. Pujolàs and S. Sibiryakov, On the Extra Mode and Inconsistency of Hor̆ava Gravity, JHEP 10 (2009) 029 [arXiv:0906.3046] [InSPIRE].

[22] M. Henneaux, A. Kleinschmidt and G. Lucena Gómez, A dynamical inconsistency of Hor̆ava gravity, Phys. Rev. D 81 (2010) 064002 [arXiv:0912.0399] [InSPIRE].

[23] I. Klebanov and A. Polyakov, AdS dual of the critical $O(N)$ vector model, Phys. Lett. B 550 (2002) 213 [hep-th/0210114] [inSPIRE].

[24] M.R. Douglas, L. Mazzucato and S.S. Razamat, Holographic dual of free field theory, Phys. Rev. D 83 (2011) 071701 [arXiv: 1011.4926] [InSPIRE].

[25] B.P. Dolan, Symplectic geometry and Hamiltonian flow of the renormalization group equation, Int. J. Mod. Phys. A 10 (1995) 2703 [hep-th/9406061] [INSPIRE]. 